\title{
A new mixed discontinuous Galerkin method for the electrostatic field
}

\author{
Abdelhamid Zaghdani ${ }^{1,2^{*}}$ and Mohamed Ezzat ${ }^{1,3}$
}

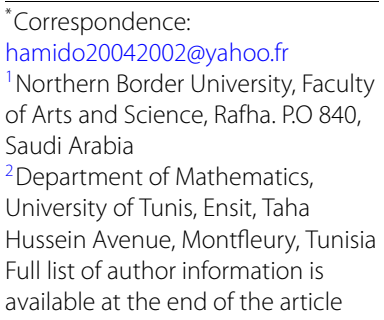

\begin{abstract}
We introduce and analyze a new mixed discontinuous Galerkin method for approximation of an electric field. We carry out its error analysis and prove an error estimate that is optimal in the mesh size. Some numerical results are given to confirm the theoretical convergence.
\end{abstract}

Keywords: Boundary value problems; Maxwell's equations; Discontinuous Galerkin formulation; A priori error estimate

\section{Introduction}

Numerical and analytical methods play an important role for solving many mathematical models arising in physics and applied sciences. Indeed, several researchers use numerical and analytical methods for solving some scientific problems (see, e.g., [1-13]). As in [1], the authors have generalized $F^{\xi}$-calculus for fractals embedding in $\mathbb{R}^{3}$, and in [12] an efficient computational technique for fractal vehicular traffic flow is presented. A new analysis of the Fornberg-Whitham equation pertaining to a fractional derivative with Mittag-Leffler-type kernel is studied by Kumar, Singh, and Baleanu [11]. Recently, Ali et al. [6] presented a new interesting two-wave version of the fifth-order Korteweg-de Vries model. Baleanu et al. [7] investigated the existence of solutions for a three-step crisis fractional integro-differential equation under some boundary conditions, and Veeresha et al. [8] presented an efficient numerical technique for the nonlinear fractional KolmogorovPetrovskii-Piskunov equation. We also cite the paper of Yang, Srivastava, and Baleanu [3], who investigated an initial-boundary value problem for the local fractional Laplace equation arising in fractal electrostatics.

During recent years, the discontinuous Galerkin method was developed and has been applied for approximating solutions of many partial differential equations, for example, Maxwell's equations [14-22], Navier-Stokes equations [23], and Poisson's equation [24]. For the time-dependent Maxwell equations, there are works of Daveau and Zaghdani [1921]. In [19] a discontinuous Galerkin scheme for the wave equation derived from Maxwell's equations with constant coefficients was discussed and analyzed, and in [22], we studied a new discontinuous Galerkin formulation for the resolution of the wave equation with variable coefficients. 
In this paper, we consider the three-dimensional Maxwell equations

$$
\begin{aligned}
& \nabla \times\left(\mu^{-1} \nabla \times u\right)=J \quad \text { in } \Omega \subset \mathbb{R}^{3}, \\
& \nabla \cdot(\varepsilon u)=0 \quad \text { in } \Omega \subset \mathbb{R}^{3},
\end{aligned}
$$

with the boundary condition $n \times u=0$ on $\partial \Omega$. Here $\mu$ is the magnetic permeability, $\varepsilon$ is the electric permittivity of the medium, and $u$ is related to the electric field $E$ by the relation $E(x, t)=\operatorname{Re}(u(x) \exp (i \omega t))$, where $\omega$ is a given nonzero frequency. We assume that $\mu$ and $\varepsilon$ are sufficiently smooth and there exist $\varepsilon_{\min }, \varepsilon_{\max }, \mu_{\min }$, and $\mu_{\max }$ such that $0<\varepsilon_{\min }<\varepsilon(x)<$ $\varepsilon_{\max }$ and $0<\mu_{\min }<\mu(x)<\mu_{\max }$ for all $x \in \Omega$. This paper is a generalization of [20], where problem (1) was studied with constant coefficients and was also studied in [25]. Here we analyze a new discontinuous Galerkin method with a symmetric principal bilinear form, but in [25] the primal formulation used to establish an a priori error estimate is no longer consistent due to the nature of the lifting operators.

To control the divergence of the electric field, we choose the mixed discontinuous Galerkin formulation. We introduce a Lagrange multiplier and consider

$$
\begin{aligned}
& \nabla \times\left(\mu^{-1} \nabla \times u\right)-\varepsilon \nabla p=J \quad \text { in } \Omega \subset \mathbb{R}^{3}, \\
& \nabla \cdot(\varepsilon u)=0 \quad \text { in } \Omega \subset \mathbb{R}^{3}, \\
& n \times u=0 \quad \text { in } \partial \Omega,
\end{aligned}
$$

together with the boundary condition $p=0$ on $\partial \Omega$.

The paper is organized as follows. Firstly, we give some notations and preliminaries related to the mesh and finite element space. Secondly, we derive a discontinuous Galerkin scheme and prove that it is consistent and well posed. Thirdly, we give an a priori error estimate. In Sects. 4 and 5, we give some numerical results to confirm the expected convergence rate as a function of the mesh size and concluding remarks, respectively.

Let $\Omega$ be a polygonal domain in $\mathbb{R}^{3}$, and let $\Pi_{h}$ be a nondegenerate quasiuniform subdivision of $\Omega$ into tetrahedra, which means that

$$
\bar{\Omega}=\bigcup_{K \in \Pi_{h}} \bar{K} \text { and } K_{i} \cap K_{j}=\emptyset \text { for } i \neq j .
$$

We denote by $F_{h}^{I}$ the set of interior faces, by $F_{h}^{D}$ the set of exterior faces, and by $F_{h}$ the set of all faces of the partition. For $s>0$, let

$$
\mathbb{H}^{s}\left(\Pi_{h}\right):=\left\{v \in L^{2}(\Omega)^{3}: v_{\mid K} \in H^{s}(K), \forall K \in \Pi_{h}\right\}
$$

and

$$
\mathbb{H}^{s}\left(\nabla \times, \Pi_{h}\right):=\left\{v: v_{\mid K} \in H^{s}(K)^{3} \text { and } \nabla \times\left(v_{\mid K}\right) \in H^{s}(K)^{3}, \forall K \in \Pi_{h}\right\}
$$

Too define the average of $\nabla \times u$ and the jump of $p$ as elements of $\Pi_{K \in \Pi_{h}} L^{2}(\partial K)^{3}$ and $\Pi_{K \in \Pi_{h}} L^{2}(\partial K)$, respectively, in formulation (13), we introduce

$$
V(h)=\mathbb{H}^{1}\left(\nabla \times, \Pi_{h}\right) \quad \text { and } \quad Q(h)=\mathbb{H}^{1}\left(\Pi_{h}\right) .
$$


Finite element spaces. Let $K \in \Pi_{h}$, and let $\mathbb{P}_{k}(K)$ be the set of polynomials of degree less than or equal to $k$ on $K$. The finite element space is taken to be

$$
D_{k}=\left\{v: v_{\mid K} \in \mathbb{P}_{k}(K)^{3}, K \in \Pi_{h}\right\} .
$$

Traces operators. We give some notations for the traces of functions $v$ in $\mathbb{H}^{s}\left(\Pi_{h}\right)^{3}$ and $w$ in $\mathbb{H}^{s}\left(\Pi_{h}\right)$ with $s>\frac{1}{2}$. Let $e \in F_{h}^{I}$ be an interior face shared by two elements $K_{1}$ and $K_{2}$, and let $n_{1}$ and $n_{2}$ be the outer unit normal vectors on $e$ with respect to $K_{1}$ and $K_{2}$, respectively. For a vector function $v$ in $\mathbb{H}^{s}\left(\Pi_{h}\right)^{3}$ and a scalar function $w$ in $\mathbb{H}^{s}\left(\Pi_{h}\right)$, we denote by $v_{1}, w_{1}$ and $v_{2}, w_{2}$ the restrictions of $v, w$ to $K_{1}$ and $K_{2}$, respectively. Then we define the average, normal, and tangential jumps of $v$ and the average and normal jump of $w$ as

$$
\begin{aligned}
& \{v\}=\frac{v_{1 \mid e}+v_{2 \mid e}}{2}, \quad[v]_{N}=v_{1 \mid e} \cdot n_{1}+v_{2 \mid e} \cdot n_{2}, \quad[v]_{T}=v_{1 \mid e} \times n_{1}+v_{2 \mid e} \times n_{2}, \\
& \{w\}=\frac{w_{1 \mid e}+w_{2 \mid e}}{2}, \quad[w]_{N}=w_{1 \mid e} n_{1}+w_{2 \mid e} n_{2} .
\end{aligned}
$$

For $e \in F_{h}^{D}$, we denote by $n$ the outward unit normal vector on $e$ and define

$$
\begin{aligned}
& \{v\}=v_{\mid e}, \quad[v]_{N}=v_{\mid e} \cdot n, \quad[v]_{T}=v_{\mid e} \times n . \\
& \{w\}=w_{\mid e}, \quad[w]_{N}=w_{\mid e} n .
\end{aligned}
$$

For $K \in \Pi_{h}$, we denote by $h_{K}$ the diameter of $K$ and define as usual $h=\max _{K \in \Pi_{h}} h_{K}$. Further, in this paper, $\sigma_{a}$ and $\sigma_{c}$ are two stabilization parameters to be chosen depending on the local mesh size. We consider the same parameters as in $[18,21]$ and define $\sigma_{a}=\frac{\kappa}{\min \left(h_{K}, h_{K^{\prime}}\right)}$ in the case of interior faces, $\sigma_{a}=\frac{\kappa}{h_{K}}$ in the case of boundary faces, and $\sigma_{c}=\frac{1}{\sigma_{a}}$ with a strictly positive constant $\kappa$ (see $[18,21]$ for more details). For the other notations and spaces used in this paper, we refer to [21].

\section{Formulation of the Maxwell problem}

To derive a weak formulation of (2), we formally multiply the first equation in (2) by a test function $v$ and the second equation in (2) by a test function $\psi$. Integrating over $K$, we obtain

$$
\begin{gathered}
\int_{K} \mu^{-1}(\nabla \times u) \cdot(\nabla \times v) d x+\int_{K} p \nabla \cdot \varepsilon v d x-\int_{\partial K}\left(\varepsilon v \cdot n_{K}\right) p d s \\
-\int_{\partial K} v \cdot\left(\left(\mu^{-1} \nabla \times u\right) \times n_{K}\right) d s=\int_{K} J \cdot v d x
\end{gathered}
$$

and

$$
-\int_{K} \varepsilon u \cdot \nabla \psi d x+\int_{\partial K}\left(\varepsilon u \cdot n_{K}\right) \psi d s=0 .
$$

Note that the functions used in the previous identities are totally discontinuous on the interfaces of the partition. Then we approximate the traces of these functions by the nu- 
merical fluxes $\widehat{\mu^{-1} \nabla \times} u, \widehat{\varepsilon u}$, and $\widehat{p}$ and transform the previous identities into

$$
\begin{gathered}
\int_{K}\left(\mu^{-1} \nabla \times u\right) \cdot(\nabla \times v) d x+\int_{K} p \nabla \cdot \varepsilon v d x-\int_{\partial K}\left(\varepsilon v \cdot n_{K}\right) \widehat{p} d s \\
-\int_{\partial K} v \cdot\left(\left(\mu^{-1 \nabla \times} u\right) \times n_{K}\right) d s=\int_{K} J \cdot v d x
\end{gathered}
$$

and

$$
-\int_{K} \varepsilon u \cdot \nabla \psi d x+\int_{\partial K}\left(\widehat{\varepsilon u} \cdot n_{K}\right) \psi d s=0
$$

\subsection{Numerical fluxes}

We define the numerical fluxes face by face by adapting the numerical fluxes defined in $[18,26]$ for the curl-curl operator and in [27] for the Laplacian operator. The fluxes are defined so that they are conservative in the sense of [28], and they give rise to a consistent formulation. We consider the following definition of numerical fluxes:

- in the interior faces of the partition,

$$
\left\{\begin{array}{l}
\widehat{\mu^{-1} \nabla \times} u=\left\{\mu^{-1} \nabla \times u\right\}-\sigma_{a}[u]_{T}, \\
\widehat{\varepsilon u}=\{\varepsilon u\}-\sigma_{c}[p]_{N}, \\
\widehat{p}=\{p\}-\sigma_{a}[\varepsilon u]_{N}
\end{array}\right.
$$

- in the exterior interfaces of the partition,

$$
\left\{\begin{array}{l}
\widehat{\mu^{-1} \nabla \times} u=\left\{\mu^{-1} \nabla \times u\right\}-\sigma_{a}[u]_{T}, \\
\widehat{\varepsilon u}=\varepsilon u-\sigma_{c} p n, \\
\widehat{p}=0 .
\end{array}\right.
$$

Now integrating back by parts equation (7), we obtain

$$
\int_{K} \psi \nabla \cdot \varepsilon u d x+\int_{\partial K}\left((\widehat{\varepsilon u}-\varepsilon u) \cdot n_{K}\right) \psi d s=0
$$

\subsection{Discontinuous Galerkin formulation}

First, we remark that for all $t, v \in \Pi_{K \in \Pi_{h}} L^{2}(\partial K)^{3}$, and $\psi \in \Pi_{K \in \Pi_{h}} L^{2}(\partial K)$, we have (see [21])

$$
\begin{aligned}
\sum_{K \in \Pi_{h}} \int_{\partial K} v\left(t \times n_{K}\right) d s & =\int_{F_{h}}[v]_{T}\{t\}-\int_{F_{h}^{I}}[t]_{T}\{v\} d s, \\
\sum_{K \in \Pi_{h}} \int_{\partial K} \psi\left(v \cdot n_{K}\right) d s & =\int_{F_{h}^{I}}\left([v]_{N}\{\psi\}+[\psi]_{N}\{v\}\right) d s+\int_{F_{h}^{D}} \psi(v \cdot n) d s .
\end{aligned}
$$


Now summing identities (6) and (10) over all elements of the partition, use formulas (11), and the definition of the numerical fluxes, we get

$$
\begin{aligned}
& \int_{\Omega} \mu^{-1} \nabla \times u \nabla \times v d x-\int_{F_{h}}[v]_{T}\left(\left\{\mu^{-1} \nabla \times u\right\} d s+\int_{F_{h}} \sigma_{a}[v]_{T}[u]_{T} d s\right. \\
& \quad+\int_{\Omega} p \nabla \cdot \varepsilon v d x-\int_{F_{h}^{I}}[\varepsilon v]_{N}\{p\} d s+\int_{F_{h}^{I}} \sigma_{a}[\varepsilon v]_{N}[\varepsilon u]_{N} d s=\int_{\Omega} J \cdot v d x, \\
& \int_{\Omega} \psi \nabla \cdot \varepsilon u d x-\int_{F_{h}^{I}}[\varepsilon u]_{N}\{\psi\} d s-\int_{F_{h}^{I}} \sigma_{c}[p]_{N}[\psi]_{N} d s-\int_{F_{h}^{D}} \sigma_{c}[\psi]_{N}[p]_{N} d s=0 .
\end{aligned}
$$

Since $[u]_{T}=0$ in $F_{h}^{I}, n \times u=0$ in $F_{h}^{D}$, and $\nabla \cdot \varepsilon u=0$ in $\Omega$ for the exact solution $u$, we can introduce the quantity

$$
-\int_{F_{h}}[u]_{T}\left\{\mu^{-1} \nabla \times v\right\} d s+r \int_{\Omega}(\nabla \cdot \varepsilon u)(\nabla \cdot \varepsilon v) d x
$$

as a penalization term. Now the discontinuous Galerkin formulation is:

Find $(u, p) \in V(h) \times Q(h)$ such that

$$
\begin{cases}A(u, v)+B(v, p)=L(v), & \forall v \in V(h), \\ B(u, \psi)-C(p, \psi)=0, & \forall \psi \in Q(h),\end{cases}
$$

where $A, B$, and $C$ are the bilinear forms defined on $V(h) \times V(h), V(h) \times Q(h)$, and $Q(h) \times$ $Q(h)$ by

$$
A(u, v):=a(u, v)-J(v, u)-J(u, v)
$$

with

$$
\begin{aligned}
J(u, v):= & \int_{F_{h}}[u]_{T}\left\{\mu^{-1} \nabla \times v\right\} d s, \\
a(u, v):= & \int_{\Omega} \mu^{-1}(\nabla \times u) \cdot(\nabla \times v) d x+\int_{F_{h}} \sigma_{a}[u]_{T}[v]_{T} d s \\
& +\int_{F_{h}^{I}} \sigma_{a}[\varepsilon u]_{N}[\varepsilon v]_{N} d s+r \int_{\Omega}(\nabla \cdot \varepsilon u)(\nabla \cdot \varepsilon v) d x, \\
B(v, p):= & \int_{\Omega} p \nabla \cdot \varepsilon v d x-\int_{F_{h}^{I}}[\varepsilon v]_{N}\{p\} d s,
\end{aligned}
$$

and

$$
C(p, \psi):=\int_{F_{h}} \sigma_{c}[p][\psi] d s
$$

Note that the first term in (12) is introduced to symmetrize the principal bilinear form of the formulation, the second term in (12) is introduced to obtain a principal bilinear form coercive not only on the kernel of the bilinear form $B$ but also on the whole discrete space. This second term may not be introduced, and in this case, we prove the coercivity of $A$ on the kernel of $B$, and this property is satisfied. 


\subsection{Discrete formulation}

The finite element method for formulation (13) consists in approximating the spaces $V(h)$ and $Q(h)$ by the finite-dimensional spaces $\mathcal{V}_{h}:=D_{2}$ and $\mathcal{Q}_{h}:=D_{1}$, respectively. The discrete formulation is given by: Find $\left(u_{h}, p_{h}\right) \in \mathcal{V}_{h} \times \mathcal{Q}_{h}$ such that

$$
\begin{cases}A\left(u_{h}, v\right)+B\left(v, p_{h}\right)=L(v), & \forall v \in \mathcal{V}_{h}, \\ B\left(u_{h}, \psi\right)-C\left(p_{h}, \psi\right)=0, & \forall \psi \in \mathcal{Q}_{h} .\end{cases}
$$

In the following section, we study the well-posedness of our mixed DG formulation. First, let us define the mesh-dependent norm on the spaces $\mathcal{V}_{h}$ and $\mathcal{Q}_{h}$. For $u \in \mathcal{V}_{h}$ and $p \in \mathcal{Q}_{h}$, we set

$$
\begin{aligned}
\|u\|_{h}^{2}:= & \left\|\mu^{-\frac{1}{2}} \nabla \times u\right\|_{0, \Omega}^{2}+\left\|\sqrt{\sigma_{a}}[\varepsilon u]_{N}\right\|_{0, F_{h}^{I}}^{2}+r\|\nabla \cdot \varepsilon u\|_{0, \Omega}^{2} \\
& +\left\|\sqrt{\sigma_{a}}[u]_{T}\right\|_{0, F_{h}}^{2}+\left\|\frac{1}{\sqrt{\sigma_{a}}}\left\{\mu^{-\frac{1}{2}} \nabla \times u\right\}\right\|_{0, F_{h}}^{2}
\end{aligned}
$$

and

$$
\|p\|_{h}^{2}:=\|p\|_{0, \Omega}^{2}+\left\|\sqrt{\sigma_{c}}[p]\right\|_{0, F_{h}}^{2}+\left\|\sqrt{\sigma_{c}}\{p\}\right\|_{0, F_{h}}^{2} .
$$

It is obvious that these quantities define norms on the spaces $\mathcal{V}_{h}$ and $\mathcal{Q}_{h}$, respectively. Now we prove that the mixed DG formulation (19) is consistent, and it has only one solution.

\subsubsection{Theorem}

Under the assumptions on $\mu$ and $\varepsilon$, there exists a constant $\kappa_{0}>0$ such that for all $\kappa \geq \kappa_{0}$, problem (19) is consistent and has only one discrete solution.

Proof The consistency of problem (19) can be deduced from the derivation of the DG formulation. Indeed, after some integration by parts and using the fact that the exact solution $(u, p) \in H_{0}(\nabla \times, \Omega) \cap H\left(\nabla_{\varepsilon^{\cdot}}, \Omega\right) \times H_{0}^{1}(\Omega)$ (we refer to [21] for the definition of this space), we can prove that the exact solution of (2) satisfies formulation (19) and the consistency follows. Since problem (19) is linear finite-dimensional space, to prove the existence and uniqueness of a solution, we only have to prove that if $J=0$, then $\left(u_{h}, p_{h}\right)=(0,0)$. Letting $J=0$, setting $(v, \psi)=(u, p)$ in (19), and subtracting the second equation from the first one, we get

$$
a(u, u)-2 J(u, u)+C(p, p)=0 .
$$

Then

$$
\begin{aligned}
& \int_{\Omega} \mu^{-1}(\nabla \times u) \cdot(\nabla \times u) d x+\int_{F_{h}} \sigma_{a}[u]_{T}[u]_{T} d s+\int_{F_{h}^{I}} \sigma_{a}[\varepsilon u]_{N}[\varepsilon u]_{N} d s \\
& \quad+r \int_{\Omega}(\nabla \cdot \varepsilon u)(\nabla \cdot \varepsilon u) d x \\
& \quad-2 \int_{F_{h}}[u]_{T}\left\{\mu^{-1} \nabla \times u\right\} d s+\int_{F_{h}} \sigma_{c}[p]_{N}^{2} d s=0 .
\end{aligned}
$$


Using the Cauchy-Schwarz inequality and the boundedness of $\mu$, we deduce

$$
2 J(u, u) \leq 2 \delta \int_{F_{h}} \sigma_{a}[u]_{T}^{2} d s+\frac{2}{\delta} C \int_{F_{h}} \frac{1}{\sigma_{a}}\left|\left\{\nabla \times u^{h}\right\}\right|^{2} d s \quad \text { for any } \delta>0 .
$$

Now, we apply the following inverse inequality [14, 29]:

$$
\|q\|_{0, \partial K}^{2} \leq C \frac{1}{h_{K}}\|q\|_{0, K}^{2}, \quad \forall q \in P_{k}(K)
$$

and since $\nabla \times \mathcal{V}_{h} \subset \mathcal{V}_{h}$, we obtain

$$
\int_{F_{h}}\left|\frac{1}{\sqrt{\sigma_{a}}}\{\nabla \times v\}\right|^{2} d s \leq \frac{C}{\kappa} \int_{\Omega}|\nabla \times v|^{2} d x, \quad \forall v \in \mathcal{V}_{h}
$$

which implies that

$$
2 J(u, u) \leq 2 \delta \int_{F_{h}} \sigma_{a}[u]_{T}^{2} d s+\frac{2}{\delta} \frac{C}{\kappa} \int_{\Omega}|\nabla \times u|^{2} d x .
$$

We use the fact that $\mu$ is bounded and deduce that

$$
\begin{aligned}
a(u, u)-2 J(u, u)+C(p, p) \geq & \left(1-\frac{2 C}{\delta \kappa}\right) \int_{\Omega}\left(\nabla \times u^{h}\right)^{2} d x+r \int_{\Omega}\left(\nabla \cdot \varepsilon u^{h}\right)^{2} d x \\
& +(1-2 \delta) \int_{F_{h}} \sigma_{a}\left[u^{h}\right]_{T}^{2} d s+\int_{F_{h}^{I}} \sigma_{a}\left[\varepsilon u^{h}\right]_{N}^{2} d s \\
& +\int_{F_{h}} \sigma_{c}\left[p^{h}\right]^{2} d s .
\end{aligned}
$$

If we choose $\delta, \kappa$ such that $1-2 \delta>0$ and $1-\frac{2 C}{\delta \kappa}>0$, we obtain

$$
\begin{aligned}
& \nabla \times u=0 \quad \text { in } \Omega, \\
& \nabla \cdot \varepsilon u=0 \quad \text { in } \Omega, \\
& {[u]_{T}=0 \quad \text { in } F_{h},} \\
& {[\varepsilon u]_{N}=0 \quad \text { in } F_{h}^{I},} \\
& {[p]_{N}=0 \quad \text { in } F_{h} .}
\end{aligned}
$$

We deduce from the first and third equations that $u$ belongs to the set $H_{0}(\nabla \times 0, \Omega)$. From the second and fourth equations we deduce that $u \in H\left(\nabla_{\varepsilon} \cdot 0, \Omega\right)$. Therefore $u=0 \mathrm{ev}$ erywhere in $\Omega$. Now the fifth equation gives that $p$ belongs to the space $H_{0}^{1}(\Omega)$, and the second equation in (19) implies, after integration by parts,

$$
-\int_{\Omega} v \nabla p d x=0, \quad \forall v \in \mathcal{V}_{h},
$$

and therefore $p=0$ in $\Omega$. 


\section{A priori error estimate}

First, we study the properties of the bilinear forms $A, B$, and $C$ on the spaces $\mathcal{V}_{h} \times \mathcal{V}_{h}$, $\mathcal{V}_{h} \times \mathcal{Q}_{h}$, and $\mathcal{Q}_{h} \times \mathcal{Q}_{h}$, respectively. Using the Cauch-Schwarz inequality, the definition of $A, B, C$, and the mesh-dependent norm, we can obtain the continuity of the bilinear forms $A, B$, and $C$, and we have the following:

\subsection{Proposition}

There exists a constant $C$ independent of $h$ such that

$$
\begin{aligned}
& |A(u, v)| \leq C\|u\|_{h}\|v\|_{h}, \quad \forall u, v \in \mathcal{V}_{h}, \\
& |B(u, \psi)| \leq C\|u\|_{h}\|\psi\|_{h}, \quad \forall u \in \mathcal{V}_{h}, \forall \psi \in \mathcal{Q}_{h}, \\
& |C(p, q)| \leq C\|p\|_{h}\|q\|_{h}, \quad \forall p, q \in \mathcal{Q}_{h} .
\end{aligned}
$$

For the coercivity of the bilinear form of $A$, we know that if $A$ is coercive on the kernel of $B$, then together with an inf-sup condition, we can demonstrate some convergence results. Nevertheless, we prove the coercivity of $A$ on the whole space $\mathcal{V}_{h} \times \mathcal{V}_{h}$.

\subsection{Proposition}

There exists a constant $\alpha_{0}$ independent of $h$ such that

$$
A(u, u) \geq \alpha_{0}\|u\|_{h}^{2}, \quad \forall u \in \mathcal{V}_{h} .
$$

Proof From inequality (28), since $C(p, p)=\int_{F_{h}} \sigma_{c}[p]_{N}^{2} d s$, after subtracting $C(p, p)$ from the left side and $\int_{F_{h}} \sigma_{c}[p]^{2} d s$ from the right side, we deduce

$$
\begin{aligned}
& a(u, u)-2 J(u, u) \\
& \geq\left(1-\frac{2 C}{\delta \kappa}\right) \int_{\Omega}\left(\nabla \times u^{h}\right)^{2} d x+r \int_{\Omega}\left(\nabla \cdot \varepsilon u^{h}\right)^{2} d x \\
& \quad+(1-2 \delta) \int_{F_{h}} \sigma_{a}\left[u^{h}\right]_{T}^{2} d s+\int_{F_{h}^{I}} \sigma_{a}\left[\varepsilon u^{h}\right]_{N}^{2} d s .
\end{aligned}
$$

Since $A(u, u)=a(u, u)-2 J(u, u)$ and

$$
\begin{aligned}
\|u\|_{h}^{2}= & a(u, u)+\left\|\frac{1}{\sqrt{\sigma_{a}}}\left\{\mu^{-\frac{1}{2}} \nabla \times u\right\}\right\|_{0, F_{h}}^{2} \\
= & \int_{\Omega}\left|\mu^{-\frac{1}{2}} \nabla \times u\right|^{2} d x+\int_{F_{h}} \sigma_{a}[u]_{T}^{2} d s+\int_{F_{h}^{I}} \sigma_{a}[\varepsilon u]_{N}^{2} d s \\
& +r \int_{\Omega}|\nabla \cdot \varepsilon u|^{2} d x+\left\|\frac{1}{\sqrt{\sigma_{a}}}\left\{\mu^{-\frac{1}{2}} \nabla \times u\right\}\right\|_{0, F_{h}}^{2}
\end{aligned}
$$


and since $\mu$ is bounded, we deduce that

$$
\begin{aligned}
A(u, u)-\alpha\|u\|_{h}^{2} \geq & \left(1-\frac{2 C}{\delta \kappa}-\alpha\right) \int_{\Omega}\left(\nabla \times u^{h}\right)^{2} d x+r(1-\alpha) \int_{\Omega}\left(\nabla \cdot \varepsilon u^{h}\right)^{2} d x \\
& +(1-2 \delta-\alpha) \int_{F_{h}} \sigma_{a}\left[u^{h}\right]_{T}^{2} d s+(1-\alpha) \int_{F_{h}^{I}} \sigma_{a}\left[\varepsilon u^{h}\right]_{N}^{2} d s \\
& -\alpha\left\|\frac{1}{\sqrt{\sigma_{a}}}\left\{\mu^{-\frac{1}{2}} \nabla \times u\right\}\right\|_{0, F_{h}}^{2} .
\end{aligned}
$$

We use inequality (26) for $u$ to write

$$
\begin{aligned}
A(u, u)-\alpha\|u\|_{h}^{2} \geq & \left(1-\frac{2 C}{\delta \kappa}-\alpha-\alpha \frac{C}{\kappa}\right) \int_{\Omega}\left(\nabla \times u^{h}\right)^{2} d x+r(1-\alpha) \int_{\Omega}\left(\nabla \cdot \varepsilon u^{h}\right)^{2} d x \\
& +(1-2 \delta-\alpha) \int_{F_{h}} \sigma_{a}\left[u^{h}\right]_{T}^{2} d s+(1-\alpha) \int_{F_{h}^{I}} \sigma_{a}\left[\varepsilon u^{h}\right]_{N}^{2} d s .
\end{aligned}
$$

Now, we choose $\delta, \kappa$, and $\alpha$ such that the right side in the previous inequality is greater than zero, and the coercivity result follows immediately.

\subsection{Inf-sup condition}

This section is devoted to prove an inf-sup condition of the bilinear form $B$. The following lemma is necessary for this proof.

\subsubsection{Lemma}

For all $v \in H^{1}\left(\Pi_{h}\right)^{3}$, there exists an interpolant $R_{h} \in D_{2}$ such that

$$
\begin{aligned}
& \int_{K}\left(\nabla \cdot \varepsilon v-R_{h}\right) q_{h} d x=0, \quad \forall q_{h} \in P_{1}(K), \forall K \in \Pi_{h}, \\
& \int_{f}\left(\varepsilon v-R_{h}\right) d s=0, \quad \forall f \in F_{h} .
\end{aligned}
$$

Proof The proof follows from Theorem 1 in [30] since $\varepsilon v \in H^{1}\left(\Pi_{h}\right)^{3}$ for all $v \in H^{1}\left(\Pi_{h}\right)^{3}$ if $\varepsilon$ is sufficiently regular.

Now the inf-sup condition is given in the following theorem

\subsubsection{Theorem}

There exists a constant $\beta$ independent of $h$ such that

$$
\inf _{q \in Q_{h} \backslash\{0\}} \sup _{v \in V_{h} \backslash\{0\}} \frac{B(v, q)}{\|q\|_{h}\|v\|_{h}} \geq \beta>0
$$

Proof Let us first remark that for all $q \in \mathcal{Q}_{h}$, we have

$$
\left\|q_{h}\right\|_{0, \Omega}^{2}=\frac{1}{3}\left\|q_{h}\right\|_{0, \Omega}^{2}+\frac{1}{3}\left\|q_{h}\right\|_{0, \Omega}^{2}+\frac{1}{3}\left\|q_{h}\right\|_{0, \Omega}^{2}
$$


Using the inverse inequality (25), since $\sigma_{c}=\frac{1}{\sigma_{a}}$, we get

$$
\begin{aligned}
\left\|q_{h}\right\|_{0, \Omega}^{2} & \geq \frac{1}{3}\left\|q_{h}\right\|_{0, \Omega}^{2}+C\left\|\frac{1}{\sqrt{\sigma_{a}}}[q]\right\|_{0, F_{h}}^{2}+C\left\|\frac{1}{\sqrt{\sigma_{a}}}\{q\}\right\|_{0, F_{h}}^{2} \\
& \geq \frac{1}{3}\left\|q_{h}\right\|_{0, \Omega}^{2}+C\left\|\sqrt{\sigma_{c}}[q]\right\|_{0, F_{h}}^{2}+C\left\|\sqrt{\sigma_{c}}\{q\}\right\|_{0, F_{h}}^{2} \\
& \geq C\|q\|_{h}^{2} .
\end{aligned}
$$

Let $q_{h} \in Q_{h}$. There exists $\widetilde{v}_{h} \in H^{1}(\Omega)^{3}$ such that

$$
\nabla \cdot \varepsilon \widetilde{v}_{h}=q_{h} \quad \text { and } \quad\left\|\widetilde{v}_{h}\right\|_{1, \Omega} \leq C\left\|q_{h}\right\|_{0, \Omega} .
$$

We set $v_{h}=R_{h}\left(\widetilde{v}_{h}\right)$, where $R_{h}$ is the operator due to the previous lemma. Therefore

$$
B\left(v_{h}, q_{h}\right)=\sum_{K \in \Pi_{h}} \int_{K} q_{h} \nabla \cdot R_{h}\left(\widetilde{v}_{h}\right)=\sum_{K \in \Pi_{h}} \int_{K} q_{h} \nabla \cdot \varepsilon \widetilde{v}_{h}=\left\|q_{h}\right\|_{0, \Omega}^{2} \geq C\left\|q_{h}\right\|_{h}^{2} .
$$

As $R_{h}$ is continuous, we have $\left\|v_{h}\right\|_{h}=\left\|R_{h}\left(\widetilde{v}_{h}\right)\right\|_{h} \leq C\left\|\widetilde{v}_{h}\right\|_{1, \Omega} \leq C\left\|q_{h}\right\|_{0, \Omega} \leq C\left\|q_{h}\right\|_{h}$, and the inf-sup condition immediately follows.

Since the bilinear forms $A, B$, and $C$ are continuous, $A$ is coercive on the discrete space $\mathcal{V}_{h} \times \mathcal{V}_{h}$, and together with the inf-sup condition shown in (38), we can show the following a priori error estimate result; we refer to [21] for details.

\subsection{Theorem}

Let $\left(u_{h}, p_{h}\right)$ be the discrete solution of (19), and let $(u, p)$ be the exact solution of (2). Suppose that $u \in H^{t+1}\left(\Pi_{h}\right)^{3}$ and $p \in H^{s-1}\left(\Pi_{h}\right)$ with $t \geq 1$ and $s \geq 2$. Then

$$
\left\|u-u_{h}\right\|_{h}^{2}+\left\|p-p_{h}\right\|_{h}^{2} \leq C\left(h^{2 \min (2, t)}\|u\|_{t+1, \Pi_{h}}^{2}+h^{2 \min (2, s)-2}\|p\|_{s, \Pi_{h}}^{2}\right)
$$

with a positive constant independent of the mesh size $h$.

\section{Numerical results}

In this section, we present numerical experiments obtained for the three-dimensional problem (2). For simplicity, we suppose that the coefficients $\varepsilon$ and $\mu$ are constants, and so $\mu \equiv \varepsilon \equiv 1$. Additionally, in formulation (19), there appear two constants $r$ and $\kappa$. For the parameter $r$, there is no restriction, and every nonnegative real number may be considered. The parameter $\kappa$ t should be considered sufficiently large to guarantee the coercivity, but it cannot be chosen too large since otherwise the matrix associated with the bilinear form $A$ would be ill conditioned. Here we have chosen $r=1, \kappa=100$, and $\Omega=[0,1] \times[0,1] \times[0,1]$.

Example 1 In this example, we choose a current density $J$ so that the true solution of (2) is

$$
u(x, y, z)=\left(\begin{array}{l}
\left(y^{2}-y\right)\left(z^{2}-z\right) \exp (y z) \\
\left(x^{2}-x\right)\left(z^{2}-z\right) \exp (x z) \\
\left(y^{2}-y\right)\left(x^{2}-x\right) \exp (x y)
\end{array}\right)
$$


Table 1 Table of errors in the $L^{2}(\Omega)$ and energy norms

\begin{tabular}{lllllll}
\hline$h$ & $\left\|u-u^{h}\right\|_{L^{1}(\Omega)}$ & $\left\|u-u^{h}\right\|_{0, \Omega}$ & $\left\|u-u^{h}\right\|_{h}$ & $\left\|p-p^{h}\right\|_{L^{1}(\Omega)}$ & $\left\|p-p^{h}\right\|_{L^{2}(\Omega)}$ & $\left\|p-p^{h}\right\|_{h}$ \\
\hline 0.4367 & $0.3471 \mathrm{E}-01$ & $0.2109 \mathrm{E}-01$ & $0.1305 \mathrm{E}+00$ & $0.7633 \mathrm{E}-01$ & $0.1045 \mathrm{E}+00$ & $0.8891 \mathrm{E}+00$ \\
0.2184 & $0.6913 \mathrm{E}-02$ & $0.2540 \mathrm{E}-02$ & $0.3875 \mathrm{E}-01$ & $0.1325 \mathrm{E}-01$ & $0.1700 \mathrm{E}-01$ & $0.2229 \mathrm{E}+00$ \\
0.1733 & $0.3846 \mathrm{E}-02$ & $0.8845 \mathrm{E}-03$ & $0.2051 \mathrm{E}-01$ & $0.8522 \mathrm{E}-02$ & $0.1134 \mathrm{E}-01$ & $0.1700 \mathrm{E}+00$ \\
$9.268 \mathrm{E}-02$ & $0.1434 \mathrm{E}-02$ & $0.9734 \mathrm{E}-04$ & $0.4521 \mathrm{E}-02$ & $0.5440 \mathrm{E}-02$ & $0.7059 \mathrm{E}-02$ & $0.6283 \mathrm{E}-01$ \\
$7.703 \mathrm{E}-02$ & $0.6172 \mathrm{E}-03$ & $0.5080 \mathrm{E}-04$ & $0.3111 \mathrm{E}-02$ & $0.5277 \mathrm{E}-02$ & $0.6941 \mathrm{E}-02$ & $0.4660 \mathrm{E}-01$ \\
\hline
\end{tabular}

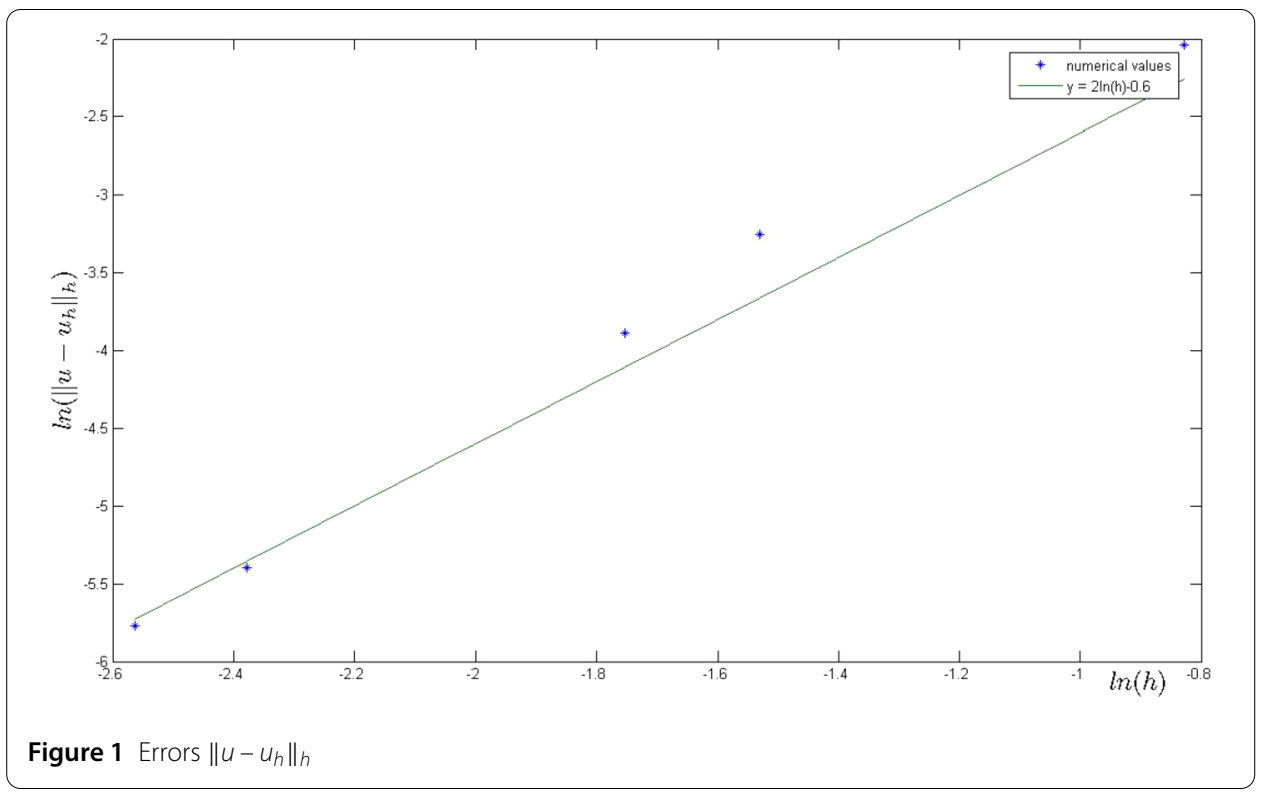

and

$$
p(x, y, z)=\left(y^{2}-y\right)\left(z^{2}-z\right)\left(x^{2}-x\right) \exp (x y z)
$$

The numerical values of errors for this example are written in Table 1. In Figs. 1 and 2, we have plotted the errors of $\left\|u-u_{h}\right\|_{h}$ and $\left\|p-p_{h}\right\|_{h}$, respectively.

Example 2 In this example, we choose the following manufactured solution $(u, p)$ of (2) together with the boundary conditions:

$$
u(x, y, z)=\left(\begin{array}{l}
\left(y^{2}-y\right)\left(z^{2}-z\right) \sin (y z) \\
\left(x^{2}-x\right)\left(z^{2}-z\right) \sin (x z) \\
\left(y^{2}-y\right)\left(x^{2}-x\right) \sin (x y)
\end{array}\right)
$$

and

$$
p(x, y, z)=\left(y^{2}-y\right)\left(z^{2}-z\right)\left(x^{2}-x\right) \sin (x y z) .
$$

We obtain the numerical values of errors in Table 2.

From the error Tables 1 and 2 and Figs. 1 and 2, we remark that the errors decrease as $h$ decreases, and we get the true solution $u, p$ with errors less than $10^{-3}$ and $10^{-2}$, respectively. These results show the convergence of the numerical solution to the exact solution 


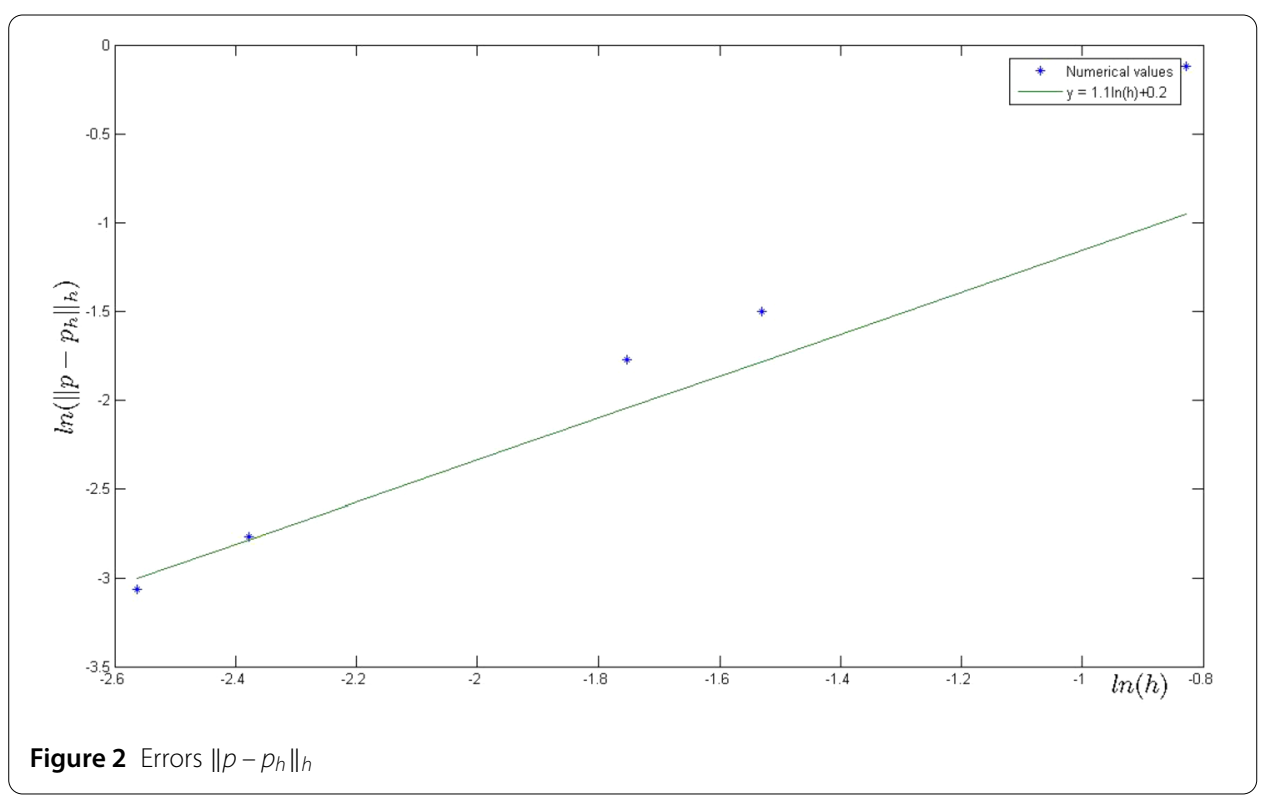

Table 2 Table of errors and order of convergence

\begin{tabular}{lllll}
\hline$h$ & $\left\|u-u^{h}\right\|_{h}$ & Order & $\left\|p-p^{h}\right\|_{h}$ & Order \\
\hline 0.4367 & $0.1102 \mathrm{E}+00$ & - & $0.7005 \mathrm{E}+00$ & - \\
0.2184 & $0.3162 \mathrm{E}-01$ & 1.80 & $0.2201 \mathrm{E}+00$ & 1.75 \\
0.1733 & $0.1808 \mathrm{E}-01$ & 2.41 & $0.1504 \mathrm{E}+00$ & 1.64 \\
$9.268 \mathrm{E}-02$ & $0.4451 \mathrm{E}-02$ & 2.23 & $0.6111 \mathrm{E}-01$ & 1.43 \\
$7.703 \mathrm{E}-02$ & $0.3052 \mathrm{E}-02$ & 2.04 & $0.4803 \mathrm{E}-01$ & 1.30 \\
\hline
\end{tabular}

according to the rate $O\left(h^{2}\right)$ for $\left\|u-u^{h}\right\|_{h}$ and $O(h)$ for $\left\|p-p^{h}\right\|_{h}$, and they confirm the theoretical result.

\section{Concluding remarks}

In this paper, we introduced a new mixed discontinuous Galerkin scheme for the approximation of the electrostatic field and analyzed and its errors. Some numerical results are given confirming the optimal convergence rates as functions of the mesh size $h$.

Acknowledgements

The authors gratefully acknowledge the approval and the support of this research at from the Deanship of Scientific Research study by the grant no. 7308-SAR-2017-1-8-F. K.S.A. Northern Border University, Arar.

Funding

This work is supported by the Deanship of Scientific Research study by the grant no. 7308-SAR-2017-1-8-F. K.S.A. Northern Border University, Arar.

Availability of data and materials

Data sharing not applicable to this paper as no data sets were generated or analyzed during the current study.

Ethics approval and consent to participate

Not applicable.

Competing interests

The authors declare that they have no competing interests.

Consent for publication

Not applicable. 
Authors' contributions

The authors contributed equally to the manuscript and typed, read, and approved the final manuscript.

\section{Author details}

${ }^{1}$ Northern Border University, Faculty of Arts and Science, Rafha. P.O 840, Saudi Arabia. ${ }^{2}$ Department of Mathematics, University of Tunis, Ensit, Taha Hussein Avenue, Montfleury, Tunisia. ${ }^{3}$ Department of Mathematics, Faculty of Science, Fayoum University, Fayoum, Egypt.

\section{Publisher's Note}

Springer Nature remains neutral with regard to jurisdictional claims in published maps and institutional affiliations.

Received: 15 May 2019 Accepted: 15 November 2019 Published online: 27 November 2019

\section{References}

1. Golmankhaneh, A.K., Golmankhaneh, A.K., Baleanu, D.: About Maxwell's equations on fractal subsets of $R^{3}$. Cent. Eur. J. Phys. 11, 863-867 (2013)

2. Alsuyuti, M.M., Doha, E.H., Ezz-Eldien, S.S., Bayoumi, B.I., Baleanu, D.: Modified Galerkin algorithm for solving multitype fractional differential equations. Math. Methods Appl. Sci. 42(5), 1389-1412 (2019)

3. Yang, X.-J., Srivastava, H.M., Baleanu, D.: Initial-boundary value problems for local fractional Laplace equation arising in fractal electrostatics. J. Appl. Nonlinear Dyn. 4(4), 349-356 (2015)

4. Khalid, A., Naeem, M.N., Ullah, Z., Ghaffar, A., Baleanu, D., Nisar, K.S., Al-Qurashi, M.M.: Numerical solution of the boundary value problems arising in magnetic fields and cylindrical shells. Mathematics 7(6), 508 (2019)

5. Golmankhaneh, A.K., Baleanu, D.: Heat and Maxwell's equations on Cantor cubes. Rom. Rep. Phys. 69, 109 (2017)

6. Ali, M., Alquran, M., Jaradat, I., et al.: Stationary wave solutions for new developed two-waves' fifth-order Korteweg-de Vries equation. Adv. Differ. Equ. 2019, 263 (2019)

7. Baleanu, D., Ghafarnezhad, K., Rezapour, S.: On a three step crisis integro-differential equation. Adv. Differ. Equ. 2019, 153 (2019)

8. Veeresha, P., Prakasha, D.G., Baleanu, D.: An efficient numerical technique for the nonlinear fractional Kolmogorov-Petrovskii-Piskunov equations. Mathematics 7, 265 (2019)

9. Ahmed, N., Rafiq, M., Baleanu, D., Rehman, M.A.: Spatio-temporal numerical modeling of auto-catalytic Brusselator model. Rom. J. Phys. 64, 110 (2019)

10. Kumar, D., Singh, J., Baleanu, D., et al.: Analysis of a fractional model of Ambartsumian equation. Eur. Phys. J. Plus 133, $259(2018)$

11. Kumar, D., Singh, J., Baleanu, D., et al.: A new analysis of Fornberg-Whitham equation pertaining to a fractional derivative with Mittag-Leffler type kernel. Eur. Phys. J. Plus 133, 70 (2018)

12. Kumar, D., Tchier, F., Singh, J., Baleanu, D.: An efficient computational technique for fractal vehicular traffic flow. Entropy 20, 259 (2018)

13. Goswami, A., Singh, J., Kumar, D., Sushila, A.: An efficient analytical approach for fractional equal width equations describing hydro-magnetic waves in cold plasma. Phys. A, Stat. Mech. Appl. 524, 563-575 (2019)

14. Houston, P., Schwab, C., Suli, E.: Discontinuous hp-finite element methods for advection diffusion- reaction problems. SIAM J. Numer. Anal. 39(6), 2133-2163 (2002)

15. Hiptmair, R., Moiola, A., Perugia, I.: Trefftz discontinuous Galerkin methods for acoustic scattering on locally refined meshes. Appl. Numer. Math. 79, 79-91 (2014)

16. Hiptmair, R., Moiola, A., Perugia, I., Schwab, C.: Approximation by harmonic polynomials in star-shaped domains and exponential convergence of Trefftz hp-dGFEM. Modél. Math. Anal. Numér. 48(3), 727-752 (2014)

17. Houston, P., Perugia, I., Schötzau, D.: hp DGFEM for Maxwell's equations. In: Brezzi, F., Buffa, A., Corsaro, S., Murli, A. (eds.) Numerical Mathematics and Advanced Applications, pp. 785-794. Springer, Milano (2003)

18. Perugia, I., Schotzau, D.: The hp-local discontinuous Galerkin method for the low-frequency time-harmonic Maxwell's equations. Math. Compet. 72, 1179-1214 (2003)

19. Daveau, C., Zaghdani, A.: A hp-discontinuous Galerkin method for the time-dependent Maxwell's equations. A priori error estimate. J. Appl. Math. Comput. 30, 1-8 (2009)

20. Daveau, C., Zaghdani, A.: Mixed discontinuous Galerkin method for the three-dimensional electrostatic problem. Int. J. Pure Appl. Math. 69(4), 357-387 (2011)

21. Zaghdani, A.: Formulations discontinues de Galerkin pour les équations de Maxwell (2006). Thèse de l'Université de Paris Sud

22. Zaghdani, A., Mohamed, M.E., El-Maghrabi, A.I.: A discontinuous Galerkin method for the wave equation: a hp a priori error estimate. J. Appl. Sci. 17, 81-89 (2017)

23. Girault, V., Rivière, B., Wheeler, M.F.: A discontinuous Galerkin method with nonoverlapping domain decomposition for the Stokes and Navier-Stokes problems. Math. Comput. 74, 53-84 (2005)

24. Prudhomme, S., Pascal, F., Oden, J.T., Romkes, A.: Review of a priori estimation for discontinuous Galerkin method. TICAM, University of Texas at Austin 27 (2000)

25. Houston, P., Perugia, I., Schotzau, D.: Mixed discontinuous Galerkin approximation of the Maxwell operator. SIAM J. Numer. Anal. 42(1), 434-459 (2004)

26. Castillo, P., Cockburn, B., Perugia, I., Schotzau, D.: An a priori error analysis of the local discontinuous Galerkin method for elliptic problems. SIAM J. Numer. Anal. 38(5), 1676-1706 (2000)

27. Cockburn, B., Kanschat, G., Perugia, l., Schotzau, D.: Superconvergence of the local discontinuous Galerkin method for elliptic problems on Cartesian grid. SIAM J. Numer. Anal. 39(1), 264-285 (2001)

28. Arnold, D.N., Brezzi, F., Cockburn, B., Marini, L.D.: Unified analysis of discontinuous Galerkin methods for elliptic problems. SIAM J. Numer. Anal. 39(5), 1749-1779 (2002)

29. Schwab, C.: p- and hp-FEM - Theory and Application to Solid and Fluid Mechanics. Oxford University Press, Oxford (1998)

30. Christian, D.: Construction of an interpolant operator: application to the three-dimensional electrostatic problem Appl. Math. Lett. 22(11), 1685-1689 (2009) 